\title{
A NOTE ON INDEPENDENT VARIABLES FOR RESTRICTED THREE-BODY PROBLEMS
}

\author{
(Research Note)
}

\author{
T. A. HEPPENHEIMER \\ Dept. of Aerospace Engineering, The University of Michigan, Ann Arbor, Mich., U.S.A.
}

(Received 23 July, 1970)

Abstract. In studies of the elliptic restricted three-body problem, the true anomaly of the motion of the primaries is often used as the independent variable. The equations of motion then show invariancy in form from the circular case. It is of interest whether other independent variables exist, such that the invariant form of the equations is maintained. It is found that true anomaly is the only such variable.

In the elliptic restricted three-body problem, it is conventional to use true anomaly $f$ as the independent variable in the equations of motion. For the classical or circular restricted problem, this variable is identical with the mean anomaly, $l$. Szebehely and Giacaglia (1964) have shown that use of $f$ in the elliptic problem results in invariancy of the form of the equations of motion.

In the circular problem, the equations of motion may be written:

$$
z^{\prime \prime}+2 i z^{\prime}=\operatorname{grad}_{z} \Omega(z) .
$$

Here $z=x+i y$ defines position in the (uniformly) rotating coordinate system, where the following quantities are taken as unity for purposes of normalization: mean motion of the primaries, the sum of their masses, the semimajor axis of their orbit. Primes denote differentiation with respect to $l$.

Then,

$$
\begin{aligned}
\Omega(z) & =\frac{1}{2}\left(x^{2}+y^{2}\right)+\frac{1-\mu}{r_{1}}+\frac{\mu}{r_{2}}+\frac{1}{2} \mu(1-\mu) \\
r_{1}^{2} & =(x-\mu)^{2}+y^{2} ; \quad r_{2}^{2}=(x+1-\mu)^{2}+y^{2} ;
\end{aligned}
$$

$\mu$ is the mass of the smaller primary.

Also, $\operatorname{grad}_{z} \equiv(\partial / \partial x)+i(\partial / \partial y)$. Separating Equation (1) into real and imaginary parts given the coupled equations of motion in $x$ and $y$.

Now consider the elliptic problem. According to Szebehely and Giacaglia, the equations of motion may be written:

$$
\zeta^{\prime \prime}+2 i \zeta^{\prime}=\operatorname{grad}_{\zeta} \omega(\zeta)
$$

where primes denote differentiation with respect to $f$. Here $\zeta=\xi+$ in defines position in a (nonuniformly) rotating system; $\xi$ and $\eta$ are pulsating coordinates, normalized with respect to $r$, the variable distance between primaries. Note that $r=\left(1-e^{2}\right) \times$ $(1+e \cos f)^{-1}$ where $e$ is eccentricity of the orbit of the primaries. The same three 
quantities as in the circular case are taken as unity, and the potential function $\omega(\zeta)$ is given by

$$
\omega(\zeta)=(1+e \cos f)^{-1} \Omega(\zeta),
$$

where $\Omega(\zeta)$ is formally equivalent to $\Omega(z)$. Note that the primaries are fixed in this coordinate system, having locations $(\xi, \eta)=(\mu, 0)$ and $(1-\mu, 0)$, respectively.

It now is of interest whether a more general independent variable $\theta$ exists such that the form of the equations of motion in the elliptic problem is still preserved. It will then still be possible to write the equations of motion in the form

$$
\frac{\mathrm{d}^{2} \varrho}{\mathrm{d} \theta^{2}}+2 i \frac{\mathrm{d} \varrho}{\mathrm{d} \theta}=\operatorname{grad}_{\varrho} P(\varrho),
$$

where $P$ is a scalar function of the pulsating complex position $\varrho$, which is normalized with respect to $r=r(\theta)$. It is not required that $P(\varrho)=g(\theta) \Omega(\varrho)$.

Following Szebehely (1967) this problem will be studied.

Consider a nonuniformly rotating coordinate system whose angular position, in inertial space, is defined by the independent variable $\theta$. In inertial space, the equations of motion are written

$$
\frac{\mathrm{d}^{2} Z}{\mathrm{~d} l^{2}}=-m_{1} k^{2} \frac{Z-Z_{1}}{R_{1}^{3}}-m_{2} k^{2} \frac{Z-Z_{2}}{R_{2}^{3}},
$$

where $Z=X+i Y, R_{j}=\left(X-X_{j}\right)^{2}+\left(Y-Y_{j}\right)^{2}$ and $\left(X_{j}, Y_{j}\right)$ is the location of the $j$ th primary. Introducing the rotating system, the equations of motion become

$$
\frac{\mathrm{d}^{2} z}{\mathrm{~d} l^{2}}+2 \frac{\mathrm{d} \theta}{\mathrm{d} l} \frac{\mathrm{d} z}{\mathrm{~d} l}=-k^{2} m_{1} \frac{z-z_{1}}{R_{1}^{3}}-k^{2} m_{2} \frac{z-z_{2}}{R_{2}^{3}}+z\left(\frac{\mathrm{d} \theta}{\mathrm{d} l}\right)^{2}-i z \frac{\mathrm{d}^{2} \theta}{\mathrm{d} l^{2}},
$$

where $z$ is the position in the rotating system, $Z=z e^{i \theta}$.

Now let $\varrho=z / r$ and $r=r(\theta)$. Transform from coordinates $z, l$ to coordinates $\varrho, \theta$ :

$$
\begin{aligned}
r\left(\frac{\mathrm{d} \theta}{\mathrm{d} t}\right)^{2}( & \left.\frac{\mathrm{d}^{2} \varrho}{\mathrm{d} \theta^{2}}+2 i \frac{\mathrm{d} \varrho}{\mathrm{d} \theta}\right)+\varrho\left(\frac{\mathrm{d}^{2} r}{\mathrm{~d} l^{2}}-r\left(\frac{\mathrm{d} \theta}{\mathrm{d} l}\right)^{2}\right)+\left(\frac{\mathrm{d} \varrho}{\mathrm{d} \theta}+i \varrho\right) \times \\
\times\left(r \frac{\mathrm{d}^{2} \theta}{\mathrm{d} l^{2}}+2 \frac{\mathrm{d} r}{\mathrm{~d} l} \frac{\mathrm{d} \theta}{\mathrm{d} l}\right) & =-\frac{k^{2}}{r^{2}}\left(m_{1} \frac{\varrho-\varrho_{1}}{\tilde{r}_{1}^{3}}+m_{2} \frac{\varrho-\varrho_{2}}{\tilde{r}_{2}^{3}}\right),
\end{aligned}
$$

where $\tilde{r}_{j}^{2}=\left(1 / r^{2}\right)\left(\varrho-\varrho_{j}\right)$. The location $\varrho_{j}$ of the $j$ th primary is not constant in the rotating system, but is a function of $\theta$.

In order that Equation (7) be expressible in the form of Equation (4), it is necessary (but not sufficient) that

$$
r \frac{\mathrm{d}^{2} \theta}{\mathrm{d} l^{2}}+2 \frac{\mathrm{d} r}{\mathrm{~d} l} \frac{\mathrm{d} \theta}{\mathrm{d} l}=0 .
$$

Equation (8) is equivalent to the condition

$$
r^{2} \frac{\mathrm{d} \theta}{\mathrm{d} l}=\mathrm{const},
$$


which is precisely the condition for conservation of angular momentum in the motion of the primaries. Taking the constant as unity gives the conclusion, $\theta=f$. This, then, is the most general independent variable for which the equations of motion, Equation (5), are expressible in the form (4). The sufficiency of the condition (8) then is established from consideration of the equations of motion for the two primaries.

In conclusion, it may be stated that the use of the independent variable $f$ in the elliptic problem, or of the variable $l$ in the circular problem, is justifiable on the grounds that no other independent variable would allow expression of the equations of motion in the 'standard form', Equation (4). Thus, use of, for example, eccentric anomaly $E$, $\cos f$, etc. are ruled out.

\section{Acknowledgements}

The author would like to thank N. X. Vinh for helpful comments, and the NASA Spacecraft Center (Lunar Mission Analysis Branch) for their support.

\section{References}

Szebehely, V. G.: 1967, Theory of Orbits, Academic Press, New York, pp. 589-594.

Szebehely, V. and Giacaglia, G. O.: 1964, 'On the Elliptic Restricted Problem', Astron. J. 69, 230. 